\title{
Modelo de regresión de Cox para análisis de supervivencia en pacientes con cáncer de mama en la provincia de Manabí, Ecuador
}

\author{
Cox regression model for survival analysis in patients with breast cancer in the province of \\ Manabí, Ecuador
}

Cecilia Bucheli Giler ${ }^{1}$, Daniel Fabricio Alarcón Cano², Karime Montes Escobar ${ }^{3}$

DOI. 10.21931/RB/2021.06.03.24

Resumen: El cáncer de mama es un problema de salud pública, que ha venido incrementándose, ubicándose como el cáncer de mayor incidencia en las mujeres, su repercusión en la población en general ocupaba el segundo lugar a nivel mundial, siendo la neoplasia maligna más frecuente en la población femenina y en relación a los decesos por cáncer y afecta con más frecuencia a los países del primer mundo y en vías de desarrollo. Estudio observacional, descriptivo y retrospectivo realizado a pacientes diagnosticados con cáncer de mama en el Hospital Oncológico de Manabí, Ecuador. La supervivencia global y libre de enfermedad a seis años se estableció a partir del tiempo trascurrido desde el diagnóstico hasta la ocurrencia de un evento o fecha del último contacto, con límite a diciembre de 2015. De los 403 pacientes, los límites de edad fueron 15 y 90 años, con media de 56.08 años. Se considero el tamaño del tumor, donde (T1) representa el 26.55\%, (T2) representaron 45.66\%, la supervivencia global fue de $80 \%$ a 6 años. Los pacientes en etapas avanzadas tuvieron menores probabilidades de supervivir con un porcentaje del 43\%. Con el modelo de regresión de Cox, fue posible demostrar asociación estadísticamente significativa entre el tamaño del tumor y la supervivencia. El estudio demuestra que los pacientes en etapas avanzadas tienen menores probabilidades de supervivir, por lo que es imperativo que se continúen esfuerzos en promoción de la salud hasta conseguir que la detección sea en etapas curables.

Palabras clave: Modelo de Riesgos Proporcionales de Cox, supervivencia, cáncer de mama, Ecuador.

Abstract: Breast cancer is a public health problem, which has been increasing, ranking as cancer with the highest incidence in women, its impact on the general population ranked second worldwide, being the most frequent malignant neoplasm in the female population and concerning deaths from cancer and affects more frequently the first world and developing countries. Observational, descriptive, and retrospective study carried out in patients diagnosed with breast cancer at the Oncological Hospital of Manabí, Ecuador. The sixyear global and disease-free survival was established from the time elapsed from the diagnosis to the occurrence of an event or the date of the last contact, with a limit to December 2015. Of the 403 patients, the age limits were 15 and 90 years, with a mean of 56.08 years. The tumor size was considered, where (T1) represents $26.55 \%$, (T2) represented $45.66 \%$, the overall survival was $80 \%$ at 6 years. Patients in advanced stages were less likely to survive with a percentage of $43 \%$. With the Cox regression model, it was possible to demonstrate a statistically significant association between tumor size and survival. The study shows that patients in advanced stages are less likely to survive, so efforts in health promotion must continue until detection is in curable stages.

Key words: Cox Proportional Hazards Model, survival, breast cancer, Ecuador.

\section{Introducción}

Según cifras de Globocan, el cáncer de mama es un problema de salud pública, que desde el año 2012 ha venido incrementándose, ubicándose como el cáncer de mayor incidencia en las mujeres ${ }^{1}$; para el año 2018 su repercusión en la población en general ocupaba el segundo lugar a nivel mundial, alcanzando el 11.6\%; siendo la neoplasia maligna más frecuente en la población femenina y en relación a los decesos por cáncer, ocupa el cuarto lugar con un 6.6\%. El cáncer de mama afecta con más frecuencia a los países del primer mundo y en vías de desarrollo, la edad con más recurrencia a la mortalidad es en mujeres de 60 a 74 años².

Para la Organización mundial de la salud, el desafío de la problemática de esta enfermedad es detectarla a tiempo. En Ecuador, según registros del Ministerio de Salud Pública, hasta junio de 2018 se realizaron 1.287 nuevas atenciones con diagnóstico de cáncer de mama, de las cuales 1.254, corresponden a mujeres representando el $97,6 \%$ de los casos presentados por esta patología, de acuerdo a datos obtenidos del Registro
Diario Automatizado de Consultas y Atenciones Ambulatorias (RDACAA 2018) Y Plataforma de Registro de Atención en Salud (PRAS 2018)

Seguidamente el Instituto de Estadísticas y Censos (INEC), en el año 2015, Manabí era la provincia que ocupaba el cuarto puesto a nivel nacional en decesos provocados por el cáncer de mama y el tercer puesto a nivel nacional en tasa de mortalidad por la misma enfermedad 4 . En Manabí el cáncer de mama ocupa el primer lugar entre los diferentes tipos de cáncer que existen; siendo esta una de las principales provincias de nuestro país, donde se encuentran estadísticas que puntúan al cáncer de mama como la principal causa de mortalidad femenina. En total, 235 casos en el 2018, que representan el 14,28\%, según estadística emitida por Solca (Sociedad de Lucha contra el Cáncer) $)^{5}$.

Con base en lo anterior se han desarrollado Modelos de Riesgos Proporcionales de Cox, para predecir la supervivencia de cáncer de mama, que han conllevado a estimar la super-

\footnotetext{
${ }_{1}$ Maestría en Estadística, Instituto de Posgrado, Universidad técnica de Manabí, Portoviejo, Ecuador.

${ }^{2}$ Docencia e investigación, Solca Manabí, Ecuador

${ }^{3}$ Departamento de Matemáticas y Estadística. Instituto de Ciencias Básicas. Universidad Técnica de Manabí, Portoviejo, Ecuador y Departamento de Estadística. Universidad de Salamanca, Salamanca, España.
} 
vivencia personalizada a largo plazo en función de las características demográficas del paciente, los factores tumorales y tratamiento entregado ${ }^{6}$; este modelo permite identificar los factores clínicamente significativos asociados con el cáncer de mama y esto puede ayudar a predecir el riesgo de enfermedad, por lo tanto sirve para obtener una evaluación adecuada de los factores clínicos relevantes para el pronóstico del cáncer, lo que contribuye particularmente en la selección de eficientes estrategias terapéuticas ${ }^{7}$

Es por ello que el presente estudio tiene como objetivo realizar un análisis de supervivencia en pacientes con cáncer de mama de la Provincia de Manabí, mediante el Modelo de Riesgos proporcionales de Cox que permita determinar la supervivencia a los 6 años de los pacientes diagnosticados con la enfermedad y su relación con las variables como edad, género y estadio del tumor, sobrevida y meses de tratamiento, que proporcionen información útil a la toma de decisiones del médico con el paciente.

\section{Métodos}

El presente trabajo es un estudio Transversal, Retrospectivo, Descriptivo, Analítico y observacional; comprendido durante el periodo 2010 hasta 2015 en el Hospital Oncológico "Dr. Julio Villacreses Colmont"; de la Sociedad de Lucha contra el cáncer SOLCA Manabí, Ecuador. Los pacientes con cáncer de mama en este periodo ascendieron a 403, que fueron objetos del análisis de supervivencia. El estudio se realizó utilizando una matriz de información sobre los pacientes con cáncer de mama, obtenida del departamento de estadística, de SOLCA, Manabí, donde se elaboró una tabla de análisis descriptivo para un examen lo más completo posible sobre el cáncer de mama, sin buscar ni causas ni consecuencias de éste. Se midió las características de manera porcentual y se observó la configuración y los procesos que componen esta enfermedad, sin detenerse a valorarlos.

Se realizó un análisis de la matriz de información, sobre las variables o características de los pacientes con cáncer de mama, consiguiendo priorizarlas en los pacientes que reunieron las características como: género, edad, estadio del tumor en relación al tamaño del mismo, la sobrevida y meses de tratamiento constituyendo esta última en la variable respuesta, en sentido de dar una mejor utilidad para el análisis de supervivencia; en ellas se analizó la relación con características clínicas o covariables que intervienen en el tiempo hasta la ocurrencia del evento o no, es decir el tiempo de censura. Este análisis de supervivencia general se efectuó mediante el programa RStudio versión 3.2.2. ${ }^{8}$.

Seguidamente se efectuó un análisis de supervivencia mediante el Modelo de Riesgos Proporcionales de Cox, donde se desarrolló el concepto de probabilidad marginal, una función de probabilidad que se caracteriza por los coeficientes de $\beta$, que miden los efectos de las covariables en la función de tasa de falla? .

El modelo de Riesgos Proporcionales de Cox, constituye un modelo multivariado que puede ponderar el efecto de una serie de covariables, variables explicativas, predictores, factores de riesgo, variables de confusión ${ }^{10}$, como también de variables cualitativas o cuantitativas sobre un desenlace dicotómico en función del tiempo y que puede definir al individuo en estudio mediante la ecuación ${ }^{11}$ :

$$
\lambda\left(t ; Z_{i}(t)\right)=\lambda_{0}(t) e^{\beta^{\prime} Z i(t)} \quad \text { ó } \quad \lambda_{i}(t)=\lambda_{0}(t) e^{x i(t) \beta}
$$

La finalidad del Modelo de Riesgos Proporcionales de Cox es facilitar un método de análisis de supervivencia sin necesidad de especificar o estimar una función de riesgo basal, mediante el desarrollo del concepto de probabilidad marginal, una función de probabilidad que solo depende de los coeficientes del modelo; al usar este modelo, el resultado se expresa por medio de una estadística llamada hazard ratio (HR), que es una división de riesgo (h) entre grupos y se define como la probabilidad de tener el desenlace en un periodo de tiempo determinado, donde hay la posibilidad de que uno de los grupos llegue antes al evento de interés al compararlo con otro grupo $^{11}$

El Modelo de Riesgos Proporcionales de Cox se basa en considerar la prueba de significación que será la variable de agrupación o de tratamiento, la misma cuyo efecto se probará con la prueba de Wald, que proporciona un valor de $\mathrm{p}$; además se considera la obtención del estimador del efecto, que es el Hazard ratio, y la obtención del intervalo de confianza de 95\% para el Hazard ratio, que permite evaluar la significancia estadística de la relación de las variables del estudio y sus efectos en el desenlace del evento de interés; en este sentido el primer supuesto que debe cumplirse será sobre los datos censurados que no están relacionados con la probabilidad que ocurra el evento, es decir, que los individuos que no llegan al evento, no abandonaron el estudio por razones relacionadas a la variable de agrupación, este supuesto se corrobora investigando las posibles razones por las que abandonaron el estudio, y el segundo supuesto seria que las curvas de supervivencia para cada uno de los estratos deben tener funciones de riesgos que sean proporcionales en el tiempo entre las etapas del problema estudiado, aplicándose a las variables cuantitativas y ordinales, en donde se logre evidenciar los riesgos con incrementos constantes de un valor a otro y que siga la misma dirección ${ }^{11}$. Este análisis de supervivencia se efectuó mediante el programa SPSS versión $25^{12}$.

Esta investigación no buscó lograr un estudio profundo sobre el Cáncer de mama en el aspecto de la medicina científica; lo que se pretendió fue lograr un análisis de supervivencia a la enfermedad de acuerdo con las variables intervinientes en el estudio en función a la tasa de muerte.

\section{Resultados}

De los 403 pacientes analizados, se observó que el $98.76 \%$, son mujeres y el $1.24 \%$ son hombres, los límites de edad fueron 15 y 90 años, con media de 56.08 años. El $45.66 \%$, presentan un tamaño del tumor T2 y el $16.63 \%$, en T4. El $30.27 \%$, tienen menos de un año padeciendo la enfermedad y el $4.96 \%$ más de cinco años. El $87.10 \%$, estaban vivos y el 12.90\% fallecieron (Tabla 1).

$$
H R=\frac{\frac{\text { Sucesos ocurridos en el instante t en el grupo 1 }}{\text { Sujeto en riesgo en el instante t en el grupo 1 }}}{\frac{\text { Sucesos ocurridos en el instante t en el grupo 2 }}{\text { Sujeto en riesgo en el instante t en el grupo 2 }}}=\frac{h 1}{h 2}
$$




\begin{tabular}{|c|c|c|c|}
\hline \multicolumn{4}{|c|}{ DESCRIPCION DE VARIABLES } \\
\hline VARIABLE & DESCRIPCIÓN & CODIFICACIÓN & $\%$ \\
\hline \multirow[t]{2}{*}{ Género } & \multirow[t]{2}{*}{ Género } & Femenino (1) & 98,76 \\
\hline & & Masculino (2) & 1,24 \\
\hline \multirow[t]{7}{*}{ Edad } & \multirow[t]{7}{*}{ Edad en años } & $15-30$ años & 2,23 \\
\hline & & 31-40 años & 12,16 \\
\hline & & $41-50$ años & 23,33 \\
\hline & & $51-60$ años & 26,55 \\
\hline & & $61-70$ años & 17,12 \\
\hline & & $71-80$ años & 11,41 \\
\hline & & $81-90$ años & 7,2 \\
\hline \multirow[t]{2}{*}{ Sobrevida } & \multirow[t]{2}{*}{ Sobrevida } & Vivo (0) & 87,1 \\
\hline & & Muerto (1) & 12,9 \\
\hline \multirow{4}{*}{$\begin{array}{c}\text { Estadío del } \\
\text { Tumor }\end{array}$} & \multirow[t]{4}{*}{ TNM } & Tamaño 1 (1) & 26,55 \\
\hline & & Tamaño 2 (2) & 45,66 \\
\hline & & Tamaño 3 (3) & 11,17 \\
\hline & & Tamaño 4 (4) & 16,63 \\
\hline \multirow[t]{6}{*}{ Meses } & \multirow[t]{6}{*}{ Meses de Tratamiento } & $0-12$ & 30,27 \\
\hline & & $13-24$ & 21,84 \\
\hline & & $24-36$ & 18,86 \\
\hline & & $37-48$ & 17,37 \\
\hline & & $49-60$ & 6,7 \\
\hline & & $61-72$ & 4,96 \\
\hline
\end{tabular}

Tabla 1. Descripción de las Variables.

Se procedió a realizar el análisis de supervivencia general, considerando el tiempo de censura y el tiempo de fallas, ubicándose a la variable meses de tratamiento como la variable respuesta del presente estudio, la misma que se mide en meses considerando la fecha del primer diagnóstico hasta la fecha del fin del estudio o la ocurrencia del evento de interés que sería la muerte del paciente con cáncer de mama. El mayor tiempo de supervivencia de los 52 pacientes que llegaron al evento, es de 40 meses para 15 pacientes (Figura 1 ).

En el estudio del tiempo de supervivencia en los pacientes con cáncer de mama se evidenciaron observaciones incompletas o parciales, es decir censuras múltiples de tipo I a la derecha (tiempo censurado); en donde el inicio del tiempo de supervivencia se realizó en diferentes fechas y a la fecha fin de estudio no se observó el evento de interés (muerte) en 351 pacientes; los mismos que no abandonaron el estudio por razones relacionadas con el tratamiento o la variable de agrupación, ya que algunos pacientes fallecieron después de la fecha fin de estudio. Entre 0 a 20 meses existe la mayor cantidad de pacientes que no llegaron al evento de interés (muerte) (Figura 2).

La Tabla 2, indica los valores de beta (B) que se encuentran asociados al hazard ratio y resultan positivos para los estadios del tumor (T3 y T4), estos estadios según los resultados re- presentan los principales factores de riesgo de la enfermedad, esto significa, que los pacientes con estadio del tumor (T3), con un 95\% de confianza (IC95\%:2.841-56.051) tienen 12.6 veces el riesgo de haber fallecido en el mes 72 de seguimiento, en relación con el estadio del tumor T1, y los pacientes con estadio del tumor T4 (IC95\%:2.902-52.900), tienen 12.4 veces el riesgo de haber fallecido en el mes 72 de seguimiento en relación con el estadio del tumor T1. Seguidamente el estadístico chi cuadrado modificado de Wald es significativo con valores $p>0.001$, para los tamaños del tumor T3 y T4 respectivamente, el resto de covariables como edad y género resultan con un valor no significativo en cuanto al riesgo de la enfermedad, por lo tanto no contribuyen con la ocurrencia del evento de interés; lo que significa que los pacientes que llegan con un tamaño del tumor en categoría T3 y T4 tienen menor tiempo de supervivencia al cáncer de mama sin incidir el género y la edad del paciente.

La curva de supervivencia global (Figura 3), en la que se representa gráficamente cómo van llegando al desenlace los 403 casos analizados conforme pasa el tiempo. La probabilidad de supervivencia global a 6 años fue de $80 \%$, esto significa que para el mes 72 de seguimiento, el $80 \%$ de los pacientes continúa con vida. El tiempo medio de supervivencia está cerca a los 54 meses.

Las curvas de supervivencia ajustadas por edad y género para cada uno de los estadios de la enfermedad reflejan cómo va incrementando la mortalidad en los pacientes en los tamaños del tumor (T1, T2, T3 y T4) de la enfermedad. Es claro que los pacientes en tamaño (T3 y T4) viven menos que los pacientes en estadios menos avanzados. Para el mes 72 cerca del $57 \%$ de los pacientes en estadio 3 y 4 habrán fallecido mientras que más del $90 \%$ de los pacientes con estadio I continúan con vida (Figura 4).

La figura 5, muestra la curva para los estadios del tumor T1, T2, T3 Y T4, donde puede verse que los estadios T3 y T4 se incrementan con mayor proporción en los intervalos del tiempo de manera que a mayor tamaño del estadio del tumor, mayor es el riesgo de supervivencia al cáncer de mama.

\section{Discusión}

En relación a los hallazgos encontrados, el Modelo de Riesgos Proporcionales de Cox ha sido de gran importancia para el presente estudio, ya que genera una descripción precisa y clara sobre la incidencia de las variables en el análisis de supervivencia del cáncer mama, considerando la variable respuesta al tiempo transcurrido desde la fecha del primer diagnóstico del paciente, hasta la fecha fin de estudio u ocurrencia del evento del interés (muerte), frente a covariables como sobrevida, estadio del tumor, edad y género; estos resultados guardan relación con lo que señala (11) que el modelo multivariante de Riesgos Proporcionales de Cox, es muy útil en el campo de la salud, cuando se trata de enfermedades graves como el cáncer, ya que el exponente de Beta o hazard ratio proporciona la significancia de la covariable más representativa en el análisis de supervivencia y así se obtiene un análisis que ayuda a la toma de decisiones sobre el tratamiento de la enfermedad entre el médico tratante con el paciente que la padece.

Así mismo los resultados del presente estudio guardan relación con lo que manifiesta (7), que el modelo de Riesgos Proporcionales de Cox genera una evaluación adecuada de los factores clínicos relevantes para el pronóstico del cáncer, lo que contribuye particularmente en la selección de eficientes 


\section{T.Superv.}

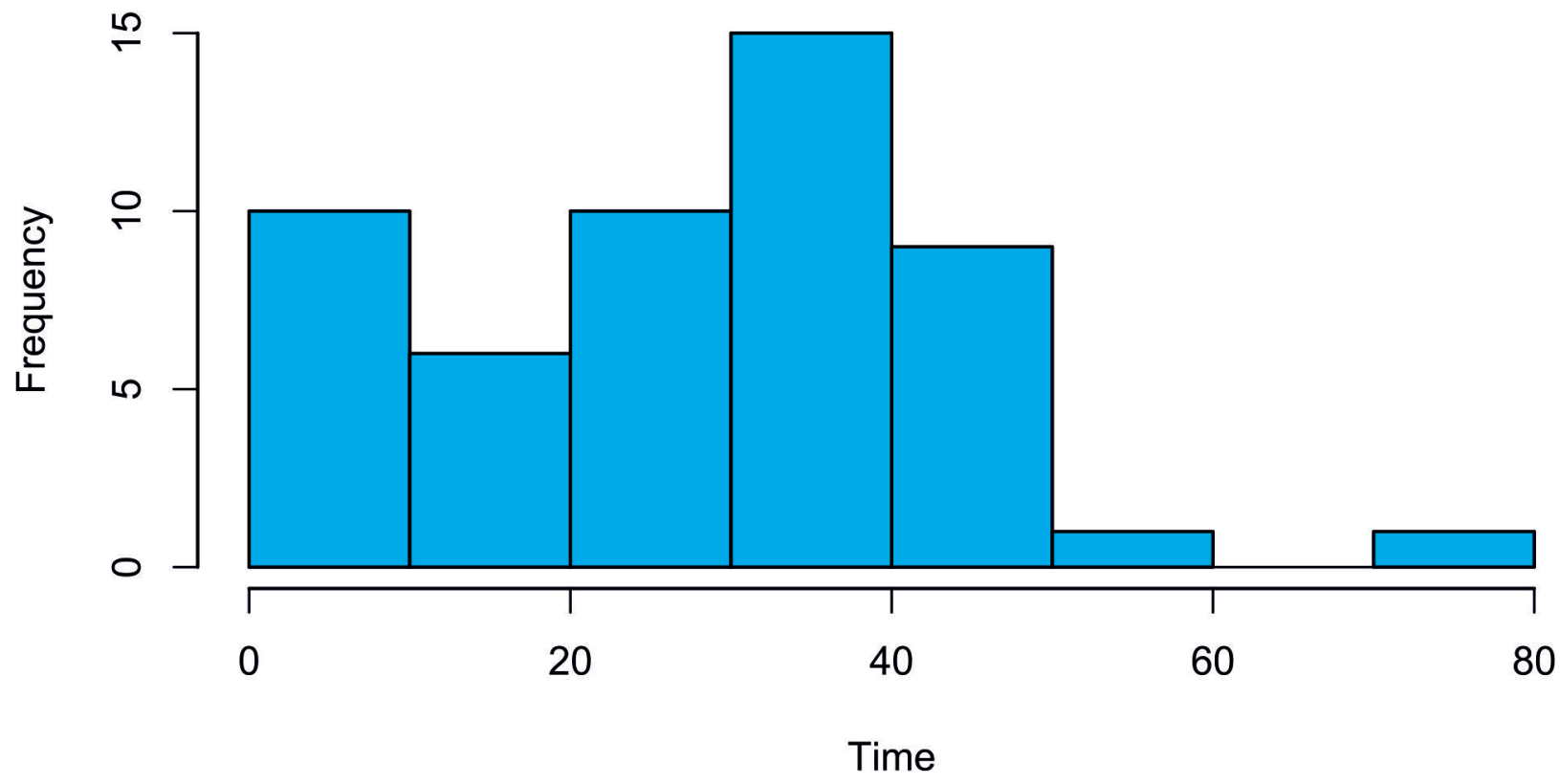

Figura 1. Tiempo de supervivencia.

\section{T.Superv.}

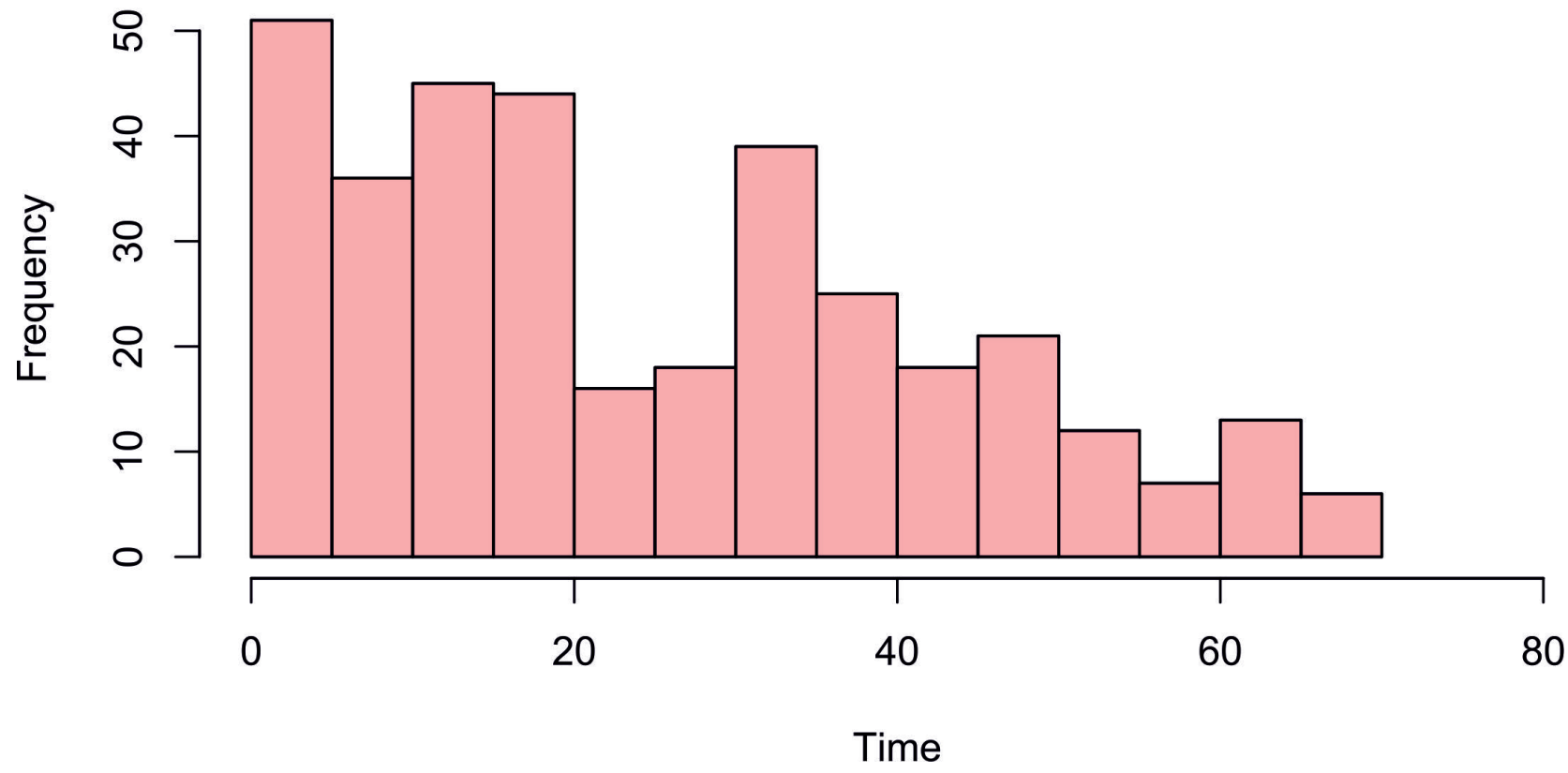

Figura 2. Tiempo censurado.

estrategias terapéuticas; pero este no concuerda con (2) ya que los pacientes con menor edad presentaran una mejor supervivencia global al cáncer de mama.

Por lo tanto en lo que se refiere a la variable edad no se pudo demostrar relación con la supervivencia, lo que tampoco coincide con estudios realizados (13), a 1113 pacientes con cáncer de mama en un periodo de estudio de cinco y diez años donde el aumento de la edad en el momento del diagnóstico se asoció con un mayor riesgo de muerte en los pacientes, así mismo en la investigación hecha por (14), a 767 pacientes con cáncer de mama, en donde se analizó la edad con la supervivencia, este encontró que los pacientes más jóvenes y mayores parecen tener una supervivencia más corta al cáncer de mama, sin embargo en este estudio no hay significancia en esta covariable edad, y por consiguiente el riesgo de que ocurra el evento no discrimina la edad del paciente.

La supervivencia global encontrada en el presente trabajo, en 6 años fue del 80\%, semejante a la reportada en el estudio de (15), que se efectuó a 119 pacientes con cáncer de mama atendidas durante el 2010 al 2015 en el Hospital Militar de Especialidades de la Mujer y Neonatología en la Ciudad de México, donde la supervivencia global se situó en $81 \%$. En lo que 


\begin{tabular}{|l|r|r|r|r|r|r|r|r|}
\hline & B & SE & Wald & df & Sig. & Exp(B) & \multicolumn{2}{|c|}{$95,0 \%$ Cl para } \\
& & & & & & & \multicolumn{2}{|c|}{ Exp(B) } \\
\cline { 5 - 9 } & & & & & & & & \\
\hline TNM & & & 24,22 & 3 & 0 & & & \\
\hline TNM(1) & 1,306 & 0,753 & 3,007 & 1 & 0,08 & 3,691 & 0,844 & 16,153 \\
\hline TNM(2) & 2,535 & 0,761 & 11,1 & 1 & 0 & 12,62 & 2,841 & 56,051 \\
\hline TNM(3) & 2,517 & 0,741 & 11,55 & 1 & 0 & 12,39 & 2,902 & 52,9 \\
\hline Género & - & 423,82 & 0,001 & 1 & 0,98 & 0 & 0 & \\
& 10,69 & & & & & & & \\
\hline Edad & 0,001 & 0,009 & 0,009 & 1 & 0,93 & 1,001 & 0,983 & 1,019 \\
\hline
\end{tabular}

Tabla 2. Factores predictores de supervivencia de los pacientes con cáncer de mama.

\section{Función de supervivencia en la media de covariables}

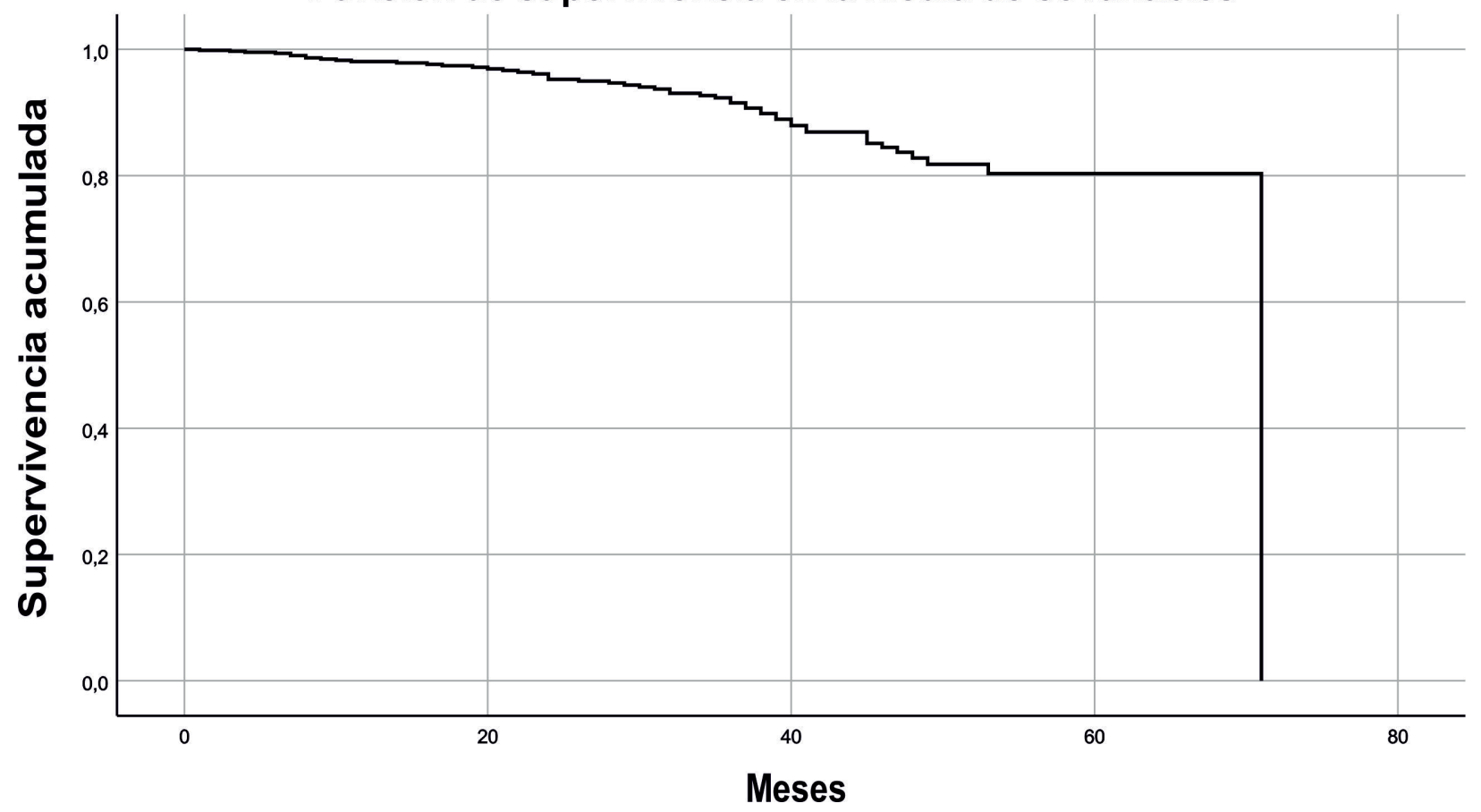

Figura 3. Curva de supervivencia ajustada por los cofactores.

no concuerda con la investigación citada, es que en esta no se pudo demostrar que la supervivencia se relaciona con otras variables como estadio del tumor (tamaño), ya que precisamente es la variable significativa relacionada con la supervivencia en los pacientes con cáncer de la presente investigación, donde tamaño del tumor T1 y T2, demostraron que a menor tamaño del tumor, mayor supervivencia a la enfermedad.

\section{Conclusiones}

En Ecuador los reportes de supervivencia son pocos, por ello se realiza esta investigación la cual significa un gran logro y esfuerzo entre instituciones, teniendo en cuenta los reportes de supervivencia a 6 años. Se demostró en este estudio, que los pacientes diagnosticados en etapas avanzadas son aquellos que su probabilidad de sobrevida es mucho menor, con base en lo anterior cabe resaltar que es de gran importancia seguir investigando en pro de la salud, esto con el fin de detectar a tiempo y en etapas curables a los pacientes.

\section{Agradecimientos}

Este estudio fue apoyado por el Departamento de Matemáticas y Estadística. Instituto de Ciencias Básicas. Los autores desean agradecer a SOLCA Portoviejo por su apoyo y facilitar los datos. 
Función de supervivencia para patrones 1 - 4

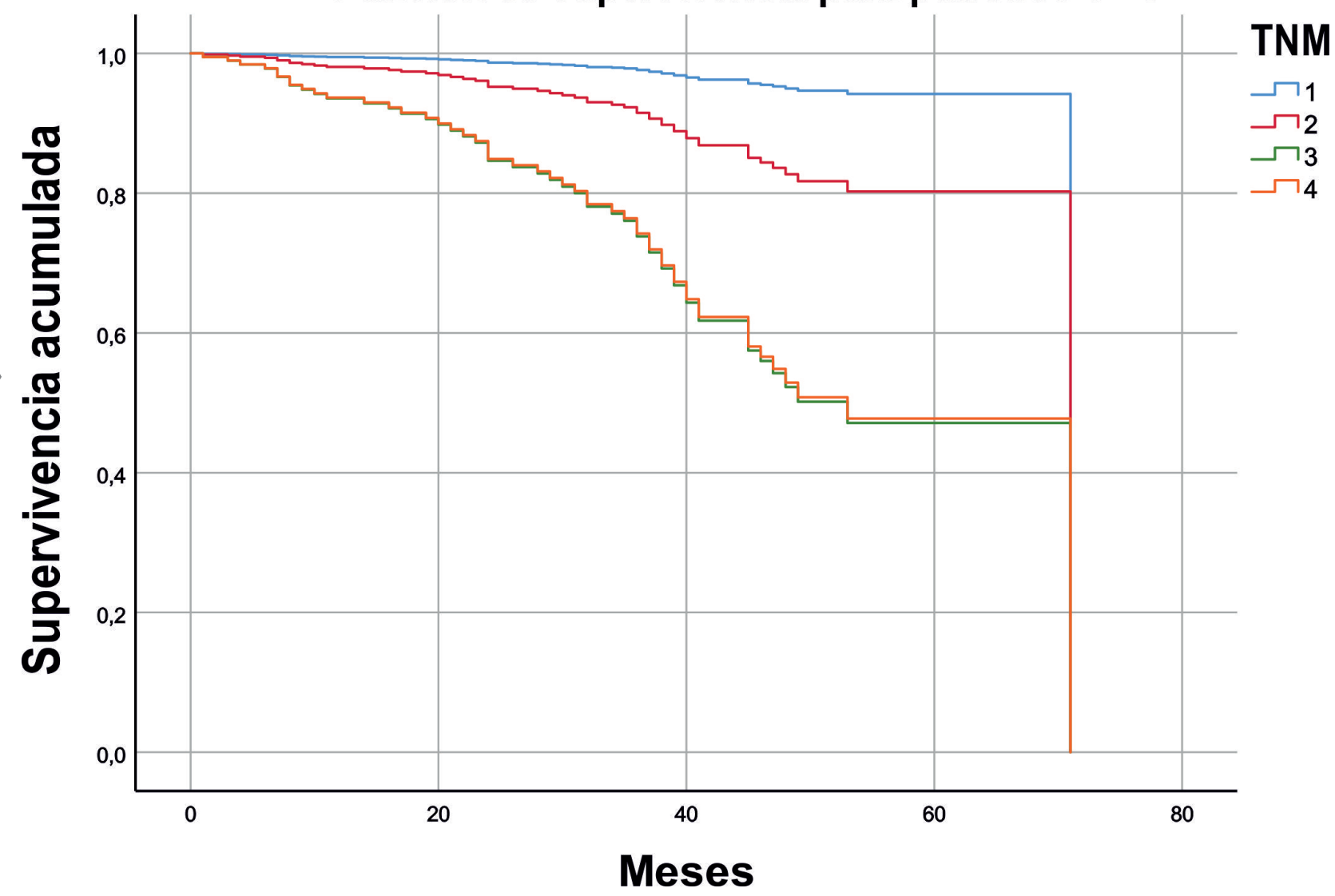

Figura 4. Curva de supervivencia por estadio de la enfermedad.

\section{Función LML para patrones 1 - 4}

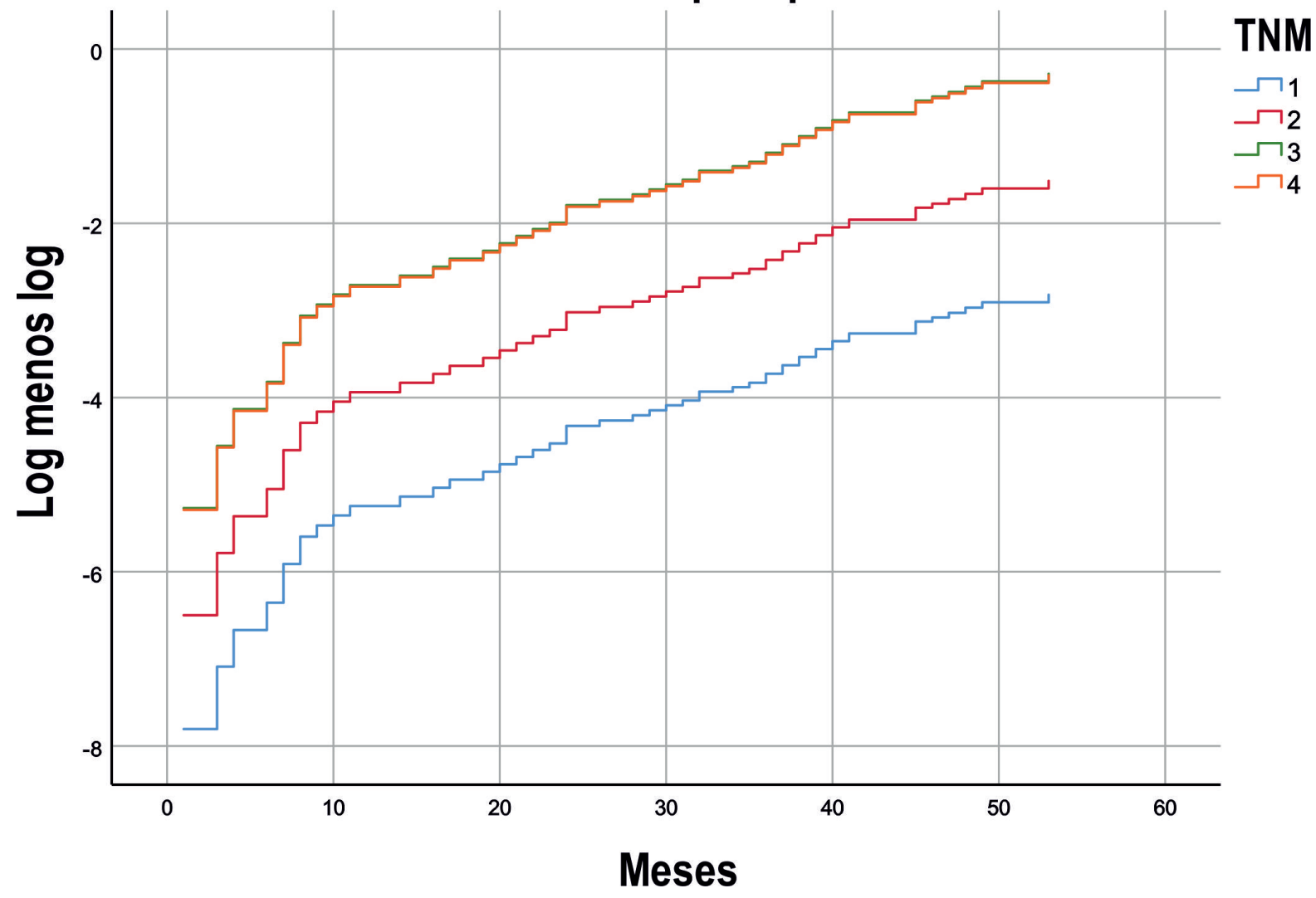

Figura 5. Evaluación de la función de riesgos proporcionales. 


\section{Referencias bibliográficas}

1. Ministerio de Salud Pública. Estrategia Nacional para la atención integral del cáncer en el Ecuador. (2017).

2. Ulloa, P., Ronquillo, S. \& Sánchez, K. Sobrevida en pacientes con cáncer de mama según su inmunohistoquímica experiencia del Instituto Oncológico Nacional - Sociedad de Lucha Contra el Cáncer, Guayaquil Ecuador. Rev. Medica Sinerg. 5, e542 (2020).

3. Ministerio de Salud Publica del Ecuador. Instructivo para el llenado del Automatizado de Consultas y Atenciones Ambulatorias (RDACAA). Minist. Salud Pública Ecuador 10-60 (2013).

4. Arreaga, A. \& Torres, L. Visualización geográfica de la tasa de mortalidad por cáncer de mama, cérvix y de próstata en el Ecuador. (2017)

5. Risco, J. Nosotros - REVISTA DE MANABÍ.. Se dieron 'un toque' para prevenir el cáncer de mama. (2019).

6. Asare, E. A. et al. Development of a model to predict breast cancer survival using data from the National Cancer Data Base. Surg (United States) 159, 495-502 (2016).

7. Tang, W. et al. Screening of Clinical Factors Related to Prognosis of Breast Cancer Based on the Cox Proportional Risk Model. J. Comput. Biol. 27, 1-10 (2020).

8. Team, R. r studio - Google Académico. RStudio: integrated development for R. RStudio, Inc. Boston, MA (2018).

9. Rebaza, D. "MODELOS SEMIPARAMÉTRICOS DE EVENTOS RECURRENTES: CASO APLICACIÓN A PACIENTES CON CANCER DE MAMA". 67 (2017).
10. Boj del Val, E. El modelo de regresión de Cox. El modelo de regresión de Cox (2017).

11. Pérez-Rodríguez, M., Rivas-Ruiz, R., Palacios-Cruz, L. \& Talavera, J. O. XXII. Del juicio clínico al modelo de riesgos proporcionales de Cox. Rev Med Inst Mex Seguro Soc 52, 430-435 (2014).

12. Spss, I. spss - Google Académico. IBM SPSS Statistics for Windows, version 25 Armonk, NY: IBM SPSS CORP. (2017).

13. Caleffi, M. et al. Breast cancer survival in Brazil: How much health care access impact on cancer outcomes? Breast 54, 155-159 (2020).

14. Balabram, D. Asociación entre edad y supervivencia en una cohorte de pacientes brasileñas con cáncer de mama operable Asociación entre edad y supervivencia en una cohorte de pacientes brasileñas con cáncer de mama operable Asociación entre edad y supervivencia en una. 31, 1732-1742 (2015).

15. Heredia, A. \& Palacios, G. Supervivencia a 5 años pos tratamiento de cáncer de mama: experiencia institucional. Ginecol. Obstet. Mex. 86, 575-583 (2018).

Received: 18 marzo 2021

Accepted: 15 julio 2021 